\title{
The non-abelian BFFT formalism for the collective coordinates quantization of the SU(2) Skyrme model
}

\author{
Wilson Oliveira*and Jorge Ananias Neto ${ }^{\dagger}$ \\ Departamento de Física, ICE \\ Universidade Federal de Juiz de Fora, 36036-330 \\ Juiz de Fora, MG, Brazil
}

\begin{abstract}
The collective coordinates expansion of the Skyrme soliton particle model gives rise to the second class constraints. We use the nonabelian BFFT formalism to convert this system into the one with only first class constraints. Choosing two different structure functions of the non-abelian algebra, we obtain simplified algebraic expressions for the first class non-abelian Hamiltonians. This result shows that the non-abelian BFFT method is, in many aspects, richer than the abelian BFFT formalism. For both of the first class Hamiltonians, we derive the Lagrangians which lead to the new theory. When one puts the extended phase space variables equal to zero, the original Skyrmion Lagrangian is reproduced. The method of the Dirac first class constraints is employed to quantize these two systems. We achieve the same spectrum, a result which confirms the consistency of the nonabelian BFFT formalism.
\end{abstract}

PACS number: 11.10.-Z, 11.10.Ef

Keywords: Skyrme model, constrained systems.

*e-mail:wilson@fisica.ufjf.br

†e-mail:jorge@fisica.ufjf.br 


\section{Introduction}

Dirac quantization of the first class constrained systems [1] has many attractive features. The quantum theory can be constructed through defining the physical states which are annihilated by the operators of the first class constraints, and then, taking the mean value of the canonical operators, we obtain the physical values. In the resulting quantum mechanics there is no Dirac bracket, and consequently, one can avoid such difficult problems as the complicated general solution of the Dirac brackets and also the factorordering problems. This problem, on the other hand, appears in the explicit representation of the canonical operators.

It is well known that the first class constraints satisfy the algebra [2]

$$
\begin{aligned}
& \left\{T_{a}, T_{b}\right\}=C_{a b}^{c} T_{c}, \\
& \left\{T_{a}, H_{0}\right\}=B_{a}^{b} T_{b},
\end{aligned}
$$

where $T_{a}$ and $T_{b}$ are the first class constraints, $C_{a b}^{c}$ and $B_{a}^{b}$ are the structure constants and $H_{0}$ is the original Hamiltonian. The physical states are obtained by imposing the condition

$$
\tilde{T}_{\alpha}|\psi\rangle_{\text {phys }}=0, \quad \alpha=1,2,
$$

where $\tilde{T}_{\alpha}$ are the operators of the first class constraints.

If the system has only the second class constraints then it is possible to convert these constraints into the ones of the first class by extending the phase space under special rules. After this, one applies the Dirac procedure described above. Batalin, Fradkin, Fradkina and Tyutin [3] developed an elegant formalism of transforming systems with the second class constraints into 
the systems which contain only the first class constraints. This is achieved with the aid of auxiliary fields which serve to extend the phase space in a convenient way to transform the second class into first class constraints. This procedure is known as the BFFT formalism. The original theory is matched when the so called unitary gauge is chosen.

In the original formulation of the BFFT formalism, the resulting first class constraints form an Abelian algebra. This is naturally the case for the systems with the linear second class constraints. Recently, Banerjee, Banerjee and Ghosh 四, have studied the non-abelian Proca model, and Oliveira and Barcelos [5] have studied the non-linear sigma model. In these works the BFFT formalism has been adapted in order that the first class constraints can form a non-abelian algebra. FFrom these examples, it might appear that the original formulation of the BFFT formalism is only addressed to the theories with linear second class constraints, while the extension of Banerjee, Banerjee and Ghosh is addressed to the non-linear ones. At same time the non-linear second class constraints for the same non-abelian Proca model and for the Skyrme model [6] have been recently studied in the context of the original BFFT formalism [4, [8]. In spite of this, it is important to emphasize that the possibility pointed out by Banerjee, Banerjee and Ghosh that one can obtain a non-abelian first class theory leads to a richer structure compared with the usual BFFT case.

The purpose of this article is to convert the second class constraints, which arise after the collective coordinates expansion of the Skyrme model into first class ones. We achieve this by applying the non-abelian BFFT formalism, and thus, employ the Dirac method of first class constraints to quantize this

\footnotetext{
${ }^{1}$ For the systems with initial first and second class constraints, the former had also to be modified in order to keep the same initial algebra, either abelian or non-abelian [9].
} 
system.

The paper is organized as follows. In Sec. 2, we give a brief outline of the usual BFFT formalism and its non-abelian extension. We also emphasize and clarify some of the particular aspects of the formalism. In Sec. 3, we apply the non-abelian BFFT formalism for the collective coordinates quantization of the SU(2) Skyrme model. We make a special choice for the structure functions, and consequently, obtain two different simplified algebras for the first class constraints and the non-abelian extended Hamiltonians. By using the Faddeev-Senjanovich path integral procedure [10] we derive the Lagrangians that lead the new theories. In Sec. 4, the spectrum of the two simplified extended theories is calculated. In Sec. 5, we present the conclusions.

\section{Brief review of the BFFT formalism and its non-abelian extension}

Let us consider a system described by a Hamiltonian $H_{0}$ in a phase space $\left(q^{i}, p^{i}\right)$ with $i=1, \ldots, N$. Here we suppose that the coordinates are bosonic (extensions to include fermionic degrees of freedom and to the continuous case can be done in a straightforward way). It is also supposed that there the system possesses only the second class constraints. Denoting them by $T_{a}$, with $a=1, \ldots, M<2 N$, we arrive at the following algebra

$$
\left\{T_{a}, T_{b}\right\}=\Delta_{a b}
$$

where $\operatorname{det}\left(\Delta_{a b}\right) \neq 0$.

As it was mentioned above, the general purpose of the BFFT formalism is to convert the second class constraints into the first class ones. This goal is achieved by introducing canonical variables, one for each of the second class 
constraint (the connection between the number of the second class constraints and the number of the new variables should be equal in order to preserve the same number of the physical degrees of freedom in the resulting extended theory). We denote these auxiliary variables by $\eta^{a}$ and assume that they satisfy the following algebra

$$
\left\{\eta^{a}, \eta^{b}\right\}=\omega^{a b} .
$$

Here $\omega^{a b}$ is a constant non-degenerate matrix $\left(\operatorname{det}\left(\omega^{a b}\right) \neq 0\right)$. The obtainment of $\omega^{a b}$ is embodied in the calculation of the resulting first class constraints which are denoted as $\tilde{T}_{a}$. Of course, these constraints depend on the new variables $\eta^{a}$, that is

$$
\tilde{T}_{a}=\tilde{T}_{a}(q, p ; \eta),
$$

and are supposed to satisfy the boundary condition

$$
\tilde{T}_{a}(q, p ; 0)=T_{a}(q, p) .
$$

In the framework of the BFFT formalism, the characteristic property of the new constraints is that they are assumed to be strongly involutive, i.e.

$$
\left\{\tilde{T}_{a}, \tilde{T}_{b}\right\}=0 .
$$

The solution of Eq. (2.5) can be achieved by considering $\tilde{T}_{a}$ expanded as

$$
\tilde{T}_{a}=\sum_{n=0}^{\infty} T_{a}^{(n)},
$$

where $T_{a}^{(n)}$ is a term of order $n$ in $\eta$. The condition of compatibility with the boundary condition (2.4) requires 


$$
T_{a}^{(0)}=T_{a}
$$

Substituting the Eq.(2.6) into (2.5) leads to a set of equations, one for each coefficient of $\eta^{n}$. We list some of them below

$$
\begin{gathered}
\left\{T_{a}, T_{b}\right\}+\left\{T_{a}^{(1)}, T_{b}^{(1)}\right\}_{(\eta)}=0 \\
\left\{T_{a}, T_{b}^{(1)}\right\}+\left\{T_{a}^{(1)}, T_{b}\right\}+\left\{T_{a}^{(1)}, T_{b}^{(2)}\right\}_{(\eta)}+\left\{T_{a}^{(2)}, T_{b}^{(1)}\right\}_{(\eta)}=0 \\
\left\{T_{a}, T_{b}^{(2)}\right\}+\left\{T_{a}^{(1)}, T_{b}^{(1)}\right\}_{(q, p)}+\left\{T_{a}^{(2)}, T_{b}\right\}+\left\{T_{a}^{(1)}, T_{b}^{(3)}\right\}_{(\eta)} \\
+\left\{T_{a}^{(2)}, T_{b}^{(2)}\right\}_{(\eta)}+\left\{T_{a}^{(3)}, T_{b}^{(1)}\right\}_{(\eta)}=0
\end{gathered}
$$

Here the notations $\{,\}_{(q, p)}$ and $\{,\}_{(\eta)}$, represent the parts of the Poisson bracket $\{$,$\} corresponding to the variables (q, p)$ and $(\eta)$, respectively. The equations above are used iteratively to obtain the corrections $T^{(n)}(n \geq 1)$. Equation (2.8) gives $T^{(1)}$. Using this result together with the Eq. (2.9), one calculates $T^{(2)}$, and so on. Since $T^{(1)}$ is linear in $\eta$ one can write it as

$$
T_{a}^{(1)}=X_{a b}(q, p) \eta^{b}
$$

where $X_{a b}$ are some new quantities. Substituting this expression into (2.8) and using (2.1) and (2.2), we obtain

$$
\Delta_{a b}+X_{a c} \omega^{c d} X_{b d}=0
$$

We notice that this equation does not define $X_{a b}$ in a unique way, because it also contains the still unknown elements $\omega^{a b}$. What is usually done is to choose $\omega^{a b}$ in such a way that the new variables are unconstrained. One 
mights mention that sometimes it is not possible to make such a choice[11]. In this case, the new variables remain constrained. Consequently, the consistency of the method requires an introduction of other new variables in order to transform these constraints into the first class ones. This may lead to an endless process. It is important to emphasize that $\omega^{a b}$ can be fixed anyway. However, even if one fixes $\omega^{a b}$, it is still not possible to obtain a unique solution for $X_{a b}$. Let us check this point. Since we are only considering bosonic coordinates 1 , $\Delta_{a b}$ and $\omega^{a b}$ are antisymmetric quantities. So, expression (2.12) includes $M(M-1) / 2$ independent equations. On the other hand, since there is no additional symmetry involving $X_{a b}$, they should represent a set of $M^{2}$ independent quantities.

In the case when $X_{a b}$ does not depend on $(q, p)$, it is easily seen that the expression $T_{a}+\tilde{T}_{a}^{(1)}$ is already strongly involutive for any choice we make and we succeed in obtaining $\tilde{T}_{a}$. If this is not so, the usual procedure is to introduce $T_{a}^{(1)}$ into Eq. (2.9) in order to calculate $T_{a}^{(2)}$ and so on. At this point one faces a problem that has been the origin of some developments of the BFFT method, including the adoption of a non-abelian constraint algebra. This occurs because we do not know a priori what is the best choice we can make to go from one step to another. Sometimes it is possible to figure out a convenient choice for $X_{a b}$ in order to obtain a first class (abelian) constraint algebra at the first stage of the process [0]. It is opportune to mention that in ref. [12], the use of a non-abelian algebra was in fact a way of avoiding to dealing with the higher orders of the iterative method. More recently, the method has been used (in its abelian version) beyond the first correction [13] but we mention that sometimes there are problems in doing this [14].

\footnotetext{
${ }^{2}$ The problem also exists for the fermionic sector.
} 
Another point of the usual BFFT formalism is that any dynamic function $A(q, p)$ (for instance, the Hamiltonian) has also to be properly modified in order to be strongly involutive with the first class constraints $\tilde{T}_{a}$. Denoting the modified quantity by $\tilde{A}(q, p ; \eta)$, we then have

$$
\left\{\tilde{T}_{a}, \tilde{A}\right\}=0 .
$$

In addition, $\tilde{A}$ has to satisfy the boundary condition

$$
\tilde{A}(q, p ; 0)=A(q, p) .
$$

The derivation of $\tilde{A}$ is similar to what has been done in getting $\tilde{T}_{a}$. Therefore, we consider an expansion of the form

$$
\tilde{A}=\sum_{n=0}^{\infty} A^{(n)},
$$

where $A^{(n)}$ is also a term of order $n$ in $\eta$ 's. Consequently, the compatibility with Eq. (2.14) requires that

$$
A^{(0)}=A \text {. }
$$

The combination of Eqs. (2.6), (2.7), (2.13), (2.15), and (2.16) gives the equations

$$
\begin{aligned}
& \left\{T_{a}, A\right\}+\left\{T_{a}^{(1)}, A^{(1)}\right\}_{(\eta)}=0 \\
& \left\{T_{a}, A^{(1)}\right\}+\left\{T_{a}^{(1)}, A\right\}+\left\{T_{a}^{(1)}, A^{(2)}\right\}_{(\eta)}+\left\{T_{a}^{(2)}, A^{(1)}\right\}_{(\eta)}=0 \\
& \left\{T_{a}, A^{(2)}\right\}+\left\{T_{a}^{(1)}, A^{(1)}\right\}_{(q, p)}+\left\{T_{a}^{(2)},\right\}+\left\{T_{a}^{(1)}, A^{(3)}\right\}_{(\eta)} \\
& \quad+\left\{T_{a}^{(2)}, A^{(2)}\right\}_{(\eta)}+\left\{T_{a}^{(3)}, A^{(1)}\right\}_{(\eta)}=0
\end{aligned}
$$


which correspond to the coefficients of the powers $0,1,2$, etc... of the variable $\eta$. It is just a matter of algebraic work to show that the general expression for $A^{(n)}$ reads as

$$
A^{(n+1)}=-\frac{1}{n+1} \eta^{a} \omega_{a b} X^{b c} G_{c}^{(n)} .
$$

where $\omega_{a b}$ and $X^{a b}$ are the inverses of $\omega^{a b}$ and $X_{a b}$, and

$$
\begin{aligned}
G_{a}^{(n)}=\sum_{m=0}^{n}\left\{T_{a}^{(n-m)}, A^{(m)}\right\}_{(q, p)}+\sum_{m=0}^{n-2}\left\{T_{a}^{(n-m)}, A^{(m+2)}\right\}_{(\eta)} \\
+\left\{T_{a}^{(n+1)}, A^{(1)}\right\}_{(\eta)} .
\end{aligned}
$$

The general prescription of the usual BFFT method to obtain the Hamiltonian is a direct use of the relations (2.15) and (2.20). This works well for the system with linear constraints. For non-linear theories, where it may be necessary to consider all orders of the iterative process, this calculation might be quite complicated. However, there is an alternative procedure that drastically simplifies the algebraic work. The basic idea is to obtain the involutive forms for the initial fields $q$ and $p$ [15]. This can be directly achieved from the previous calculation of $\tilde{A}$. Denoting such fields by $\tilde{q}$ and $\tilde{p}$ we have

$$
H(q, p) \longrightarrow H(\tilde{q}, \tilde{p})=\tilde{H}(\tilde{q}, \tilde{p}) .
$$

It is obvious that the initial boundary condition in the BFFT process, that is, the reduction of the involutive function to the original function when the new fields are set to zero, remains preserved. One can also mention that for the systems with linear constraints, the new variables $\tilde{q}$ and $\tilde{p}$ are just shifted in the auxiliary coordinate $\eta$ [16]. 
Finally, let us consider the case where the first class constraints form a non-abelian algebra, i.e.

$$
\left\{\tilde{T}_{a}, \tilde{T}_{b}\right\}=C_{a b}^{c} \tilde{T}_{c}
$$

The quantities $C_{a b}^{c}$ are the structure constants of the non-abelian algebra. These constraints are considered to satisfy the same previous conditions given by (2.3), (2.4), (2.6), and (2.7). But now, instead of Eqs. (2.8)-(2.10), we obtain

$$
\begin{aligned}
C_{a b}^{c} T_{c}= & \left\{T_{a}, T_{b}\right\}+\left\{T_{a}^{(1)}, T_{b}^{(1)}\right\}_{(\eta)} \\
C_{a b}^{c} T_{c}^{(1)}= & \left\{T_{a}, T_{b}^{(1)}\right\}+\left\{T_{a}^{(1)}, T_{b}\right\} \\
& +\left\{T_{a}^{(1)}, T_{b}^{(2)}\right\}_{(\eta)}+\left\{T_{a}^{(2)}, T_{b}^{(1)}\right\}_{(\eta)} \\
C_{a b}^{c} T_{c}^{(2)}= & \left\{T_{a}, T_{b}^{(2)}\right\}+\left\{T_{a}^{(1)}, T_{b}^{(1)}\right\}_{(q, p)} \\
& +\left\{T_{a}^{(2)}, T_{b}^{(0)}\right\}_{(q, p)}+\left\{T_{a}^{(1)}, T_{b}^{(3)}\right\}_{(\eta)} \\
& +\left\{T_{a}^{(2)}, T_{b}^{(2)}\right\}_{(\eta)}+\left\{T_{a}^{(3)}, T_{b}^{(1)}\right\}_{(\eta)+}
\end{aligned}
$$

The use of these equations is the same as before, i.e., they shall work iteratively. Equation (2.24) gives $T^{(1)}$. With this result and Eq. (2.25) one calculates $T^{(2)}$, and so on. To calculate the first correction, we assume it is given by the same general expression (2.11). Introducing it into (2.24), we now get

$$
C_{a b}^{c} T_{c}=\Delta_{a b}+X_{a c} \omega^{c d} X_{b d} .
$$

Of course, the same difficulties concerning the solutions of Eq. (2.12) also apply here, with the additional problem of choosing the appropriate struc- 
ture constants $C_{a b}^{c}$. To obtain the embedding Hamiltonian $\tilde{H}(q, p, \eta)$ one cannot use the simplified version discussed for the abelian case (embodied into Eq. (2.22) ) because the algebra is not strong involutive anymore. Thus we start from the fact that the new Hamiltonian $\tilde{H}$ and the new constraints $\tilde{T}_{a}$ satisfy the relation

$$
\left\{\tilde{T}_{a}, \tilde{H}\right\}=B_{a}^{b} \tilde{T}_{b},
$$

where the coefficients $B_{a}^{b}$ are the structure constant of the non-abelian algebra. The involutive Hamiltonian is considered to satisfy the same conditions (2.14)-(2.16). We then obtain that the general correction $H^{(n)}$ is given by a relation similar to (2.20), but now the quantities $G_{a}^{(n)}$ are given by

$$
\begin{aligned}
G_{a}^{(n)}= & \sum_{m=0}^{n}\left\{T_{a}^{(n-m)}, H^{(m)}\right\}_{(q, p)}+\sum_{m=0}^{n-2}\left\{T_{a}^{(n-m)}, A^{(m+2)}\right\}_{(\eta)} \\
& +\left\{T_{a}^{(n+1)}, A^{(1)}\right\}_{(\eta)}-B_{a}^{b} T_{c}^{(n)} .
\end{aligned}
$$

\section{The non-abelian BFFT formalism for the $\mathrm{SU}(2)$ Skyrme model}

The classical static Lagrangian of the Skyrme model is given by

$$
L=\int d^{3} r\left\{-\frac{F_{\pi}^{2}}{16} \operatorname{Tr}\left(\partial_{i} U \partial_{i} U^{+}\right)+\frac{1}{32 e^{2}} \operatorname{Tr}\left[U^{+} \partial_{i} U, U^{+} \partial_{j} U\right]^{2}\right\},
$$

where $F_{\pi}$ is the pion decay constant, $e$ is a dimensionless parameter and $\mathrm{U}$ is an $\mathrm{SU}(2)$ matrix. Performing the collective semi-classical expansion [17], substituting $\mathrm{U}(\mathrm{r})$ by $U(r, t)=A(t) U(r) A^{+}(t)$ in (2.30), where A is an $\mathrm{SU}(2)$ matrix, we obtain 


$$
L=-M+\lambda \operatorname{Tr}\left[\partial_{0} A \partial_{0} A^{-1}\right],
$$

where $\mathrm{M}$ is the soliton mass. In the hedgehog representation for $\mathrm{U}, U=$ $\exp (i \tau \cdot \hat{r} F(r))$, this mass is given by

$$
M=4 \pi \frac{F_{\pi}}{e} \int_{0}^{\infty} d x x^{2} \frac{1}{8}\left[F^{2}+2 \frac{2 \sin ^{2} F}{x^{2}}\right]+\frac{1}{2}\left[\frac{\sin ^{2} F}{x^{2}}+2 F^{\prime 2}\right],
$$

where $x$ is a dimensionless variable defined by $x=e F_{\pi} r$ and $\lambda$ is called the inertia moment written as

$$
\lambda=\frac{2}{3} \pi\left(\frac{1}{e^{3} F_{\pi}}\right) \Lambda
$$

with

$$
\Lambda=\int_{0}^{\infty} d x x^{2} \sin ^{2} F\left[1+4\left(F^{2}+\frac{\sin ^{2} F}{x^{2}}\right)\right] .
$$

The $\mathrm{SU}(2)$ matrix A can be written as $A=a^{0}+i a \cdot \tau$ with the constraint

$$
T_{1}=a^{i} a^{i}-1 \approx 0, \quad i=0,1,2,3 .
$$

The Lagrangian(2.31) can be written as a function of the $a^{i}$ as

$$
L=-M+2 \lambda \dot{a}^{i} \dot{a}^{i} .
$$

In order to identify more constraints, we calculate the momentum

$$
\pi^{i}=\frac{\partial L}{\partial \dot{a}_{i}}=4 \lambda \dot{a}^{i} .
$$

Now we can rewrite the Hamiltonian in the form 


$$
\begin{array}{r}
H_{c}=\pi^{i} \dot{a}^{i}-L=4 \lambda \dot{a}^{i} \dot{a}^{i}-L=M+2 \lambda \dot{a}^{i} \dot{a}^{i} \\
=M+\frac{1}{8 \lambda} \sum_{i=0}^{3} \pi^{i} \pi^{i} .
\end{array}
$$

Constructing the total Hamiltonian and imposing the consistency condition that constraints do not evolve in time [1] we get a new constraint

$$
T_{2}=a^{i} \pi^{i} \approx 0
$$

We observe that no further constraints are generated via this iterative procedure. The constraints $T_{1}$ and $T_{2}$ are of the second class. The matrix elements of their Poisson brackets read

$$
\Delta_{\alpha \beta}=\left\{T_{\alpha}, T_{\beta}\right\}=-2 \epsilon_{\alpha \beta} a^{i} a^{i}, \alpha, \beta=1,2
$$

where $\epsilon_{\alpha \beta}$ is the antisymmetric tensor normalized as $\epsilon_{12}=-\epsilon^{12}=-1$.

Then, the standard quantization is made where we replace $\pi^{i}$ by $-i \partial / \partial a_{i}$ in $(2.38)$, leading to

$$
H=M+\frac{1}{8 \lambda} \sum_{i=0}^{3}\left(-\frac{\partial^{2}}{\partial a_{i}{ }^{2}}\right) .
$$

Due the constraint $\sum_{i=0}^{3} a^{i} a^{i}=1$, the operator $\sum_{i=0}^{3}\left(-\frac{\partial^{2}}{\partial a_{i}{ }^{2}}\right)$ must be interpreted as the Laplacian on the three-sphere[17]. A typical polynomial wave function 17], $\frac{1}{N(l)}\left(a^{1}+i a^{2}\right)^{l}=\mid$ polynomial $\rangle$, is an eigenvector of the Hamiltonian (2.41), with the eigenvalues given by 5 .

$$
E=M+\frac{1}{8 \lambda} l(l+2), \quad l=1,2,3 \ldots .
$$

\footnotetext{
${ }^{3}$ This wave function is also eigenvector of the spin and isospin operators, written as 17 $J^{k}=\frac{1}{2}\left(a_{0} \pi_{k}-a_{k} \pi_{0}-\epsilon_{k l m} a_{l} \pi_{m}\right)$ and $I^{k}=\frac{1}{2}\left(a_{k} \pi_{0}-a_{0} \pi_{k}-\epsilon_{k l m} a_{l} \pi_{m}\right)$.
} 
To implement the extended non-abelian BFFT formalism, we introduce auxiliary coordinates, one for each of the second class constraint. Let us generally denote them by $\eta^{\alpha}$, where $\alpha=1,2$, and consider that the Poisson algebra of these new coordinates is given by

$$
\left\{\eta^{\alpha}, \eta^{\beta}\right\}=\omega^{\alpha \beta}=2 \epsilon^{\alpha \beta} ; \alpha=1,2 .
$$

From Eq. (2.27), we have

$$
2 X_{11}(x, z) X_{22}(y, z)=-2 a^{i} a^{i}+C_{12}^{1} T_{1} .
$$

After some attempts, we find that a convenient choice for these coefficients is

$$
\begin{aligned}
& X_{11}=1, \\
& X_{22}=-1, \\
& X_{12}=0=X_{21}, \\
& C_{12}^{1}=2, \\
& C_{12}^{2}=0 .
\end{aligned}
$$

Using (2.4), (2.6), (2.11), (2.43) and (2.45), the new set of constraints is found to be

$$
\begin{array}{r}
\tilde{T}_{1}=a^{i} a^{i}-1+\eta^{1}, \\
\tilde{T}_{2}=a^{i} \pi^{i}-\eta^{2}+\eta^{1} \eta^{2} .
\end{array}
$$

The first class constraint algebra is 


$$
\begin{aligned}
& \left\{\tilde{T}_{1}, \tilde{T}_{1}\right\}=0, \\
& \left\{\tilde{T}_{1}, \tilde{T}_{2}\right\}=2 \tilde{T}_{1}, \\
& \left\{\tilde{T}_{2}, \tilde{T}_{2}\right\}=0 .
\end{aligned}
$$

Next, we derive the corresponding Hamiltonian in the extended phase space. The corrections for the canonical Hamiltonian are given by Eqs. (2.20) and (2.29). With the objective to simplify the expression of the first class Hamiltonian, we chose two different algebras for the system defined by the parameters $B_{a}^{b}$ in (2.28). We have verified that possible values are

$$
B_{a}^{b}=0, \quad a, b=1,2,
$$

and

$$
B_{1}^{1}=\frac{1}{2 \lambda}, \quad B_{1}^{2}=B_{2}^{1}=B_{2}^{2}=0 .
$$

Using the inverse matrices

$$
\begin{array}{r}
\omega_{\alpha \beta}=\frac{1}{2} \epsilon_{\alpha \beta}, \\
X^{\alpha \beta}=\left(\begin{array}{cc}
1 & 0 \\
0 & -1
\end{array}\right),
\end{array}
$$

and the algebra defined by (2.49), it is possible to compute the involutive first class Hamiltonian 


$$
\begin{array}{r}
\tilde{H}=M+\frac{1}{8 \lambda} \pi^{i} \pi^{i}-\frac{1}{8 \lambda} \pi^{i} \pi^{i} \eta^{1}-\frac{1}{4 \lambda} a^{i} \pi^{i} \eta^{2}+\frac{1}{4 \lambda} a^{i} \pi^{i} \eta^{1} \eta^{2} \\
+\frac{1}{8 \lambda} a^{i} a^{i} \eta^{2} \eta^{2}-\frac{1}{8 \lambda} a^{i} a^{i} \eta^{1} \eta^{2} \eta^{2} \\
=M+\frac{1}{8 \lambda} \pi^{i} \pi^{i}\left(1-\eta^{1}\right)-\frac{1}{4 \lambda} a^{i} \pi^{i} \eta^{2}\left(1-\eta^{1}\right)+\frac{1}{8 \lambda} a^{i} a^{i} \eta^{2} \eta^{2}\left(1-\eta^{1}\right) .
\end{array}
$$

Thus, the Hamiltonian (2.53) satisfies the first class algebra

$$
\begin{aligned}
& \left\{\tilde{T}_{1}, \tilde{H}_{1}\right\}=0, \quad\left(B_{1}^{1}=B_{1}^{2}=0\right) \\
& \left\{\tilde{T}_{2}, \tilde{H}_{1}\right\}=0 . \quad\left(B_{2}^{1}=B_{2}^{2}=0\right)
\end{aligned}
$$

The other non-abelian first class Hamiltonian is given by

$$
\begin{array}{r}
\tilde{H}_{2}=\tilde{H}_{1}+\frac{1}{4 \lambda} \tilde{T}_{2} \\
=M+\frac{1}{8 \lambda} \pi^{i} \pi^{i}\left(1-\eta^{1}\right)-\frac{1}{4 \lambda} a^{i} \pi^{i} \eta^{2}\left(1-\eta^{1}\right)+\frac{1}{8 \lambda} a^{i} a^{i} \eta^{2} \eta^{2}\left(1-\eta^{1}\right) \\
+\frac{1}{4 \lambda}\left(a^{i} \pi^{i}-\eta^{2}\left(1-\eta^{1}\right)\right),
\end{array}
$$

which satisfies the first class Poisson algebra

$$
\begin{aligned}
\left\{\tilde{T}_{1}, \tilde{H}_{2}\right\} & =\frac{1}{2 \lambda} \tilde{T}_{1}, & \left(B_{1}^{1}=\frac{1}{2 \lambda}, B_{1}^{2}=0\right) \\
\left\{\tilde{T}_{2}, \tilde{H}_{2}\right\} & =0 . & \left(B_{2}^{1}=B_{2}^{2}=0\right)
\end{aligned}
$$


Here we would like to remark that, contrary the results obtained by the abelian BFFT method applied to the non-linear Lagrangian theories [7, \&], both expressions of the first class Hamiltonians (2.53) and (2.56) are finite sums. As it was emphasized in the introduction, the possibility pointed out by Banerjee, Banerjee and Ghosh to obtain non-abelian first class theories leads to a more elegant and simplified Hamiltonian structure than usual abelian BFFT case.

The next step is to look for the Lagrangian that leads to this new theory. A consistent way of doing this is by means of the path integral formalism, where the Faddeev procedure [10 has to be used. Let us identify the new variables $\eta^{\alpha}$ as a canonically conjugate pair $\left(\phi, \pi_{\phi}\right)$ in the Hamiltonian formalism,

$$
\begin{gathered}
\eta^{1} \rightarrow 2 \phi, \\
\eta^{2} \rightarrow \pi_{\phi},
\end{gathered}
$$

satisfying (2.43). Then, the general expression for the vacuum functional reads

$$
Z=N \int[d \mu] \exp \left\{i \int d t\left[\dot{a}^{i} \pi^{i}+\dot{\phi} \pi_{\phi}-\tilde{H}\right]\right\},
$$

with the measure $[d \mu]$ given by

$$
\begin{array}{r}
{[d \mu]=\left[d a^{i}\right]\left[d \pi^{i}\right][d \phi]\left[d \pi_{\phi}\right]|\operatorname{det}\{,\}|} \\
\delta\left(a^{i} a^{i}-1+2 \phi\right) \delta\left(a^{i} \pi^{i}-\pi_{\phi}+2 \phi \pi_{\phi}\right) \prod_{\alpha} \delta\left(\tilde{\Lambda}_{\alpha}\right),
\end{array}
$$

where $\tilde{\Lambda}_{\alpha}$ are the gauge fixing conditions corresponding to the first class constraints $\tilde{T}_{\alpha}$ and the term $|\operatorname{det}\{\}$,$| represents the determinant of all constraints$ 
of the theory, including the gauge-fixing ones. The quantity $\mathrm{N}$ that appears in (2.60) is an usual normalization factor. Starting from the Hamiltonian (2.53), the vacuum functional reads

$$
\begin{array}{r}
Z=N \int\left[d a^{i}\right]\left[d \pi^{i}\right][d \phi]\left[d \pi_{\phi}\right]|\operatorname{det}\{,\}| \delta\left(a^{i} a^{i}-1+2 \phi\right) \\
\delta\left(a^{i} \pi^{i}-\pi_{\phi}(1-2 \phi)\right) \prod_{\alpha} \delta\left(\tilde{\Lambda}_{\alpha}\right) \exp \left\{i \int d t \left[\dot{a}^{i} \pi^{i}+\dot{\phi} \pi_{\phi}-M\right.\right. \\
\left.\left.-\frac{1}{8 \lambda} \pi^{i} \pi^{i}(1-2 \phi)+\frac{1}{4 \lambda} a^{i} \pi^{i} \pi_{\phi}(1-2 \phi)-\frac{1}{8 \lambda} a^{i} a^{i} \pi_{\phi} \pi_{\phi}(1-2 \phi)\right]\right\} .
\end{array}
$$

Using the delta function $\delta\left(a^{i} a^{i}-1+2 \phi\right)$ and exponentiating the delta function $\delta\left[a^{i} \pi^{i}-\pi_{\phi}(1-2 \phi)\right]$ with Fourier variable $\xi$, we obtain

$$
\begin{array}{r}
Z=N \int\left[d a^{i}\right]\left[d \pi^{i}\right][d \phi]\left[d \pi_{\phi}\right][d \xi]|\operatorname{det}\{,\}| \delta\left(a^{i} a^{i}-1+2 \phi\right) \prod_{\alpha} \delta\left(\tilde{\Lambda}_{\alpha}\right) \\
\exp \left\{i \int d t \left[\dot{a}^{i} \pi^{i}+\dot{\phi} \pi_{\phi}-M-\frac{1}{8 \lambda} \pi^{j} \pi^{j} a^{i} a^{i}+\frac{1}{4 \lambda} a^{j} \pi^{j} a^{i} a^{i} \pi_{\phi}\right.\right. \\
\left.\left.-\frac{1}{8 \lambda}\left(a^{i} a^{i}\right)^{2}\left(\pi_{\phi}\right)^{2}+\xi a^{i} \pi^{i}-\xi a^{i} a^{i} \pi_{\phi}\right]\right\} .
\end{array}
$$

Integrating over $\pi_{\phi}$, we arrive at,

$$
\begin{array}{r}
Z=N \int\left[d a^{i}\right]\left[d \pi^{i}\right][d \phi][d \xi]|\operatorname{det}\{,\}| \delta\left(a^{i} a^{i}-1+2 \phi\right) \prod_{\alpha} \delta\left(\tilde{\Lambda}_{\alpha}\right) \\
\frac{1}{a^{i} a^{i}} \exp \left\{i \int d t \left[\dot{a}^{i} \pi^{i}-M-\frac{1}{8 \lambda} \pi^{j} \pi^{j} a^{i} a^{i}+\xi a^{i} \pi^{i}\right.\right. \\
\left.\left.-\frac{2 \lambda \dot{\phi} \dot{\phi}}{\left(a^{i} a^{i}\right)^{2}}+\frac{4 \lambda \dot{\phi} \xi}{a^{i} a^{i}}-2 \lambda \xi^{2}\right]\right\} .
\end{array}
$$


Performing the integration over $\pi^{i}$, we obtain

$$
\begin{array}{r}
Z=N \int\left[d a^{i}\right][d \phi][d \xi]|\operatorname{det}\{,\}| \delta\left(a^{i} a^{i}-1+2 \phi\right) \prod_{\alpha} \delta\left(\tilde{\Lambda}_{\alpha}\right) \\
\frac{1}{1-2 \phi} \sqrt{\frac{1}{1-2 \phi}} \exp \left\{i \int d t \left[-M+2 \lambda \frac{\dot{a}^{i} \dot{a}^{i}}{1-2 \phi}\right.\right. \\
\left.\left.-2 \lambda \frac{\dot{\phi} \dot{\phi}}{(1-2 \phi)^{2}}+\frac{4 \lambda}{1-2 \phi}\left(a^{i} \dot{a}^{i}+\dot{\phi}\right) \xi\right]\right\} .
\end{array}
$$

Finally, the integration over $\xi$ leads to

$$
\begin{array}{r}
Z=N \int\left[d a^{i}\right][d \phi]|\operatorname{det}\{,\}| \delta\left(a^{i} a^{i}-1+2 \phi\right) \delta\left(a^{i} \dot{a}^{i}+\dot{\phi}\right) \prod_{\alpha} \delta\left(\tilde{\Lambda}_{\alpha}\right) \\
\sqrt{\frac{1}{1-2 \phi}} \exp \left\{i \int d t \left[-M+\frac{2 \lambda}{1-2 \phi} \dot{a}^{i} \dot{a}^{i}\right.\right. \\
\left.\left.-\frac{2 \lambda}{(1-2 \phi)^{2}} \dot{\phi} \dot{\phi}\right]\right\}
\end{array}
$$

where the new $\delta$ function above came from the integration over $\xi$. We notice that it is nothing other than the derivative of constraint $\tilde{T}_{1}$. It is then just a consistency condition and does not represent any new restriction over the coordinates of the theory. From the vacuum functional (2.66), we identify the Lagrangian of the new theory

$$
L=-M+\frac{2 \lambda}{1-2 \phi} \dot{a}^{i} \dot{a}^{i}-\frac{2 \lambda}{(1-2 \phi)^{2}} \dot{\phi} \dot{\phi} .
$$

Putting the extended variables, in the phase space, $\phi$ and $\pi_{\phi}$ equal to zero, we obtain the original Skyrmion Lagrangian. This result indicates the consistency of the theory.

For the Hamiltonian (2.56) the vacuum functional is 


$$
\begin{array}{r}
Z=N \int\left[d a^{i}\right]\left[d \pi^{i}\right][d \phi]\left[d \pi_{\phi}\right]|\operatorname{det}\{,\}| \delta\left(a^{i} a^{i}-1+2 \phi\right) \\
\delta\left(a^{i} \pi^{i}-\pi_{\phi}(1-2 \phi)\right) \prod_{\alpha} \delta\left(\tilde{\Lambda}_{\alpha}\right) \exp \left\{i \int d t\left[\dot{a}^{i} \pi^{i}+\dot{\phi} \pi_{\phi}-\tilde{H}_{1}-\frac{1}{4 \lambda} \tilde{T}_{2}\right\}\right.
\end{array}
$$

Using the properties of delta functions, it is easy to see that we have obtained the same Lagrangian (2.67).

\section{The spectrum of the theory}

Here we intend to obtain the spectrum of the extended theory. We use the Dirac method of quantization for the first class constraints [1].The basic idea consists in imposing quantum mechanically the first class constraints as operator condition on the wave-functions as a way to obtain the physical subspace, i.e.,

$$
\tilde{T}_{\alpha}|\psi\rangle_{\text {phys }}=0, \quad \alpha=1,2 .
$$

The operators $\tilde{T}_{1}$ and $\tilde{T}_{2}$ are

$$
\begin{array}{r}
\tilde{T}_{1}=a^{i} a^{i}-1+\eta^{1}, \\
\tilde{T}_{2}=a^{i} \pi^{i}-\eta^{2}+\eta^{1} \eta^{2} .
\end{array}
$$

Thus, the physical states that satisfy (2.69) are

$$
\left.|\psi\rangle_{p h y s}=\frac{1}{V} \delta\left(a^{i} \pi^{i}-\eta^{2}+\eta^{1} \eta^{2}\right) \delta\left(a^{i} a^{i}-1+\eta^{1}\right) \mid \text { polynomial }\right\rangle
$$


where $V$ is the normalization factor and the ket polynomial was defined in Section 3 as, $\mid$ polynomial $\rangle=\frac{1}{N(l)}\left(a^{1}+i a^{2}\right)^{l}$. The corresponding quantum Hamiltonians of (2.53) and (2.56) will be indicated as

$$
\begin{array}{r}
\tilde{H}_{1}=M+\frac{1}{8 \lambda} \pi^{i} \pi^{i}\left(1-\eta^{1}\right)-\frac{1}{4 \lambda} a^{i} \pi^{i} \eta^{2}\left(1-\eta^{1}\right) \\
+\frac{1}{8 \lambda} a^{i} a^{i} \eta^{2} \eta^{2}\left(1-\eta^{1}\right)
\end{array}
$$

and

$$
\tilde{H}_{2}=\tilde{H}_{1}+\frac{1}{4 \lambda} \tilde{T}_{2}
$$

Thus, in order to obtain the spectrum of the theory, we take the scalar product, phys $\langle\psi|\tilde{H}| \psi\rangle_{\text {phys }}$, that is the mean value of the extended Hamiltonian, for the two quantum Hamiltonians (2.73) and (2.74). We begin with the first Hamiltonian (2.73) calculating the scalar product

$$
\begin{array}{r}
{ }_{p h y s}\left\langle\psi\left|\tilde{H}_{1}\right| \psi\right\rangle_{p h y s}= \\
\langle\text { polynomial }| \frac{1}{V^{2}} \int d \eta^{1} d \eta^{2} \delta\left(a^{i} a^{i}-1+\eta^{1}\right) \delta\left(a^{i} \pi^{i}-\eta^{2}+\eta^{1} \eta^{2}\right) \\
\tilde{H}_{1} \delta\left(\left[a^{i} \pi^{i}-\eta^{2}+\eta^{1} \eta^{2}\right) \delta\left(a^{i} a^{i}-1+\eta^{1}\right) \mid \text { polynomial }\right\rangle .
\end{array}
$$

Notice that due to the presence of the delta functions $\delta\left(a^{i} a^{i}-1+\eta^{1}\right)$ and $\delta\left(a^{i} \pi^{i}-\eta^{2}+\eta^{1} \eta^{2}\right)$ in (2.75) the scalar product can be simplified. Then, integrating over $\eta^{1}$ and $\eta^{2}$ we obtain

\footnotetext{
${ }^{4}$ The regularization of delta function squared like $\left(\delta\left(a^{i} a^{i}-1+\eta^{1}\right)\right)^{2}$ and $\left(\delta\left(a^{i} \pi^{i}-\eta^{2}+\right.\right.$ $\left.\left.\eta^{1} \eta^{2}\right)\right)^{2}$ is performed by using the delta relation, $(2 \pi)^{2} \delta(0)=\lim _{k \rightarrow 0} \int d^{2} x e^{i k \cdot x}=\int d^{2} x=$ $V$. Then, we use the parameter $\mathrm{V}$ as the normalization factor.
} 


$$
\begin{array}{r}
{ }_{\text {phys }}\left\langle\psi\left|\tilde{H}_{1}\right| \psi\right\rangle_{\text {phys }}= \\
\left\langle\text { polynomial }\left|M+\frac{1}{8 \lambda} a^{i} a^{i} \pi^{j} \pi^{j}-\frac{1}{8 \lambda} a^{i} \pi^{i} a^{j} \pi^{j}\right| \text { polynomial }\right\rangle .
\end{array}
$$

We repeat the same procedure for the quantum Hamiltonian (2.74). Taking the mean value, we have

$$
\begin{array}{r}
{ }_{\text {phys }}\left\langle\psi\left|\tilde{H}_{2}\right| \psi\right\rangle_{\text {phys }}= \\
\langle\text { polynomial }| \frac{1}{V^{2}} \int d \eta^{1} d \eta^{2} \delta\left(a^{i} a^{i}-1+\eta^{1}\right) \delta\left(a^{i} \pi^{i}-\eta^{2}+\eta^{1} \eta^{2}\right) \\
\tilde{H}_{2} \delta\left(\left[a^{i} \pi^{i}-\eta^{2}+\eta^{1} \eta^{2}\right) \delta\left(a^{i} a^{i}-1+\eta^{1}\right) \mid \text { polynomial }\right\rangle .
\end{array}
$$

Using the delta properties, we obtain the simplified scalar product for the Hamiltonian (2.74)

$$
\begin{array}{r}
{ }_{\text {phys }}\left\langle\psi\left|\tilde{H}_{2}\right| \psi\right\rangle_{\text {phys }}= \\
\left\langle\text { polynomial }\left|M+\frac{1}{8 \lambda} a^{i} a^{i} \pi^{j} \pi^{j}-\frac{1}{8 \lambda} a^{i} \pi^{i} a^{j} \pi^{j}\right| \text { polynomial }\right\rangle .
\end{array}
$$

The expression above is the same obtained for the scalar product of quantum Hamiltonian $\tilde{H}_{1}$. It is important to remark that, despite the BFFT formalism permits to have freedom to choose different first class algebras for the same second class Hamiltonian, the two expressions for the spectrum of both algebras are identical. This result shows again the consistency of the BFFT formalism.

The final Hamiltonian operator inside the kets (2.76) and (2.78) must be hermitian. Then, this Hamiltonian has to be symmetrized Following the

\footnotetext{
${ }^{5}$ In the BFFT formalism applied on the Skyrme model, the operator ordering problem appears in the expression of the non-abelian first class Hamiltonian.
} 
prescription of Weyl ordering [18 (symmetrization procedure) we can write the symmetric Hamiltonian as

$$
\tilde{H}_{\text {sym }}=\frac{1}{8 \lambda}\left[a^{i} a^{i} \pi^{j} \pi^{j}\right]_{s y m}-\frac{1}{8 \lambda}\left[a^{i} \pi^{i} a^{j} \pi^{j}\right]_{s y m},
$$

where $\left[a^{i} a^{i} \pi^{j} \pi^{j}\right]_{\text {sym }}$ and $\left[a^{i} \pi^{i} a^{j} \pi^{j}\right]_{\text {sym }}$ are defined as

$$
\begin{array}{r}
{\left[a^{i} a^{i} \pi^{j} \pi^{j}\right]_{s y m}=\frac{1}{32 \lambda}\left[a^{i}\left(a^{i} \pi^{j}+\pi^{j} a^{i}\right) \pi^{j}+\pi^{j}\left(a^{i} \pi^{j}+\pi^{j} a^{i}\right) a^{i}\right]} \\
{\left[a^{i} \pi^{i} a^{j} \pi^{j}\right]_{s y m}=\frac{1}{32 \lambda}\left[\left(a^{i} \pi^{i}+\pi^{i} a^{i}\right)\left(a^{j} \pi^{j}+\pi^{j} a^{j}\right)\right] .}
\end{array}
$$

Then, using the symmetric Hamiltonian operator $\tilde{H}_{\text {sym }}$ Eq. (2.79) both the mean values (2.76) and (2.78) are

$$
\begin{array}{r}
{ }_{p h y s}\left\langle\psi\left|\tilde{H}_{\text {sym }}\right| \psi\right\rangle_{\text {phys }}= \\
\left\langle\text { polynomial }\left|M+\frac{1}{8 \lambda}\left[a^{i} a^{i} \pi^{j} \pi^{j}\right]_{\text {sym }}-\frac{1}{8 \lambda}\left[a^{i} \pi^{i} a^{j} \pi^{j}\right]_{\text {sym }}\right| \text { polynomial }\right\rangle . \\
=\langle\text { polynomial }| M+\frac{1}{32 \lambda}\left[a^{i}\left(a^{i} \pi^{j}+\pi^{j} a^{i}\right) \pi^{j}+\pi^{j}\left(a^{i} \pi^{j}+\pi^{j} a^{i}\right) a^{i}\right] \\
\left.-\frac{1}{32 \lambda}\left[\left(a^{i} \pi^{i}+\pi^{i} a^{i}\right)\left(a^{j} \pi^{j}+\pi^{j} a^{j}\right)\right] \mid \text { polynomial }\right\rangle
\end{array}
$$

The operator $\pi^{j}$ describes a free particle and its representation on the collective coordinates space $a^{i}$ is given by

$$
\pi^{j}=-i \frac{\partial}{\partial a_{j}} .
$$

Substituting the expression (2.83) into (2.82), we obtain

$$
{ }_{\text {phys }}\left\langle\psi\left|[\tilde{H}]_{\text {sym }}\right| \psi\right\rangle_{\text {phys }}=M+\frac{1}{8 \lambda}[l(l+2)+1] .
$$


This last expression, Eq. (2.84), differs from the conventional energy eigenvalues of the Skyrme model, Eq. (2.42), by an additional constant term. Thus, using the symmetrized non-abelian BFFT Hamiltonians and employing the Dirac quantization method of first class constraints, we have obtained the Skyrmion rotational mode energy eigenvalues with a mass shift. Similar results have been also obtained by many authors[1] [19] using different procedures.

\section{Conclusions}

We have used an extension of the BFFT formalism presented by Banerjee, Banerjee and Ghosh in order to quantize the SU(2) Skyrme model. Using the non-abelian algebra, we have shown that, contrary to the results obtained by the usual abelian BFFT formalism, it is possible to construct the first class Hamiltonians that are simple finite sums. The extended Lagrangians were achieved by using the Faddeev-Senjanovich constraint path integral formalism. In the so called unitary gauge we reproduced the original Skyrmion Lagrangian. We calculate the mean energy for the two different first class Hamiltonian operators leading consistently to the same mass spectrum of the theory. Then, our results show, in some sense, that for the non-linear theory the non-abelian BFFT formalism is more adequate than the abelian formalism.

\footnotetext{
${ }^{6}$ These authors have pointed out that a mass shift can improve the usual phenomenology predicted by the Skyrme model.
} 


\section{Acknowledgments}

We would like to thank Ilya Shapiro for critical reading. This work is supported in part by FAPEMIG, Brazilian Research Council.

\section{References}

[1] P.A.M. Dirac, Proc.Roy.Soc.A257, 32 (1960). Lectures on Quantum Mechanics (Yeshiva University Press, New York, 1964).

A.Hanson, T.Regge and C.Teitelboim, Constrained Hamiltonian Systems (Academia Nazionale dei Lincei, Roma 1976).

K.Sundermeyer, Constrained Dynamics, Lectures Notes in Physics (Springer, New York, 1982), vol 169.

[2] Marc Henneaux and Claudio Teitelboim, Quantization of Gauge Systems, (Princeton University Press 1992).

[3] I.A.Batalin and I.V.Tyutin, Int.J.Mod.Phys.A6, 3255 (1991).

[4] N. Banerjee, R. Banerjee and S. Ghosh, Ann.Phys. (NY)241, 237 (1995).

[5] J.Barcelos-Neto and W.Oliveira, Phys.Rev.D56,4 2257 (1997).

W.Oliveira, PHD Thesis, UFRJ (1997).

[6] T.H.Skyrme, Proc.Roy.Soc.A260, 127 (1961).

[7] N. Banerjee, R. Banerjee, and S. Ghosh, Nucl.Phys.B417, 257 (1994).

[8] W.Oliveira and J. Ananias Neto, Int. J. Mod. Phys.A, vol.12,no.27, 4895 (1997).

[9] W. T. Kim and Y. -J. Park, Phys.Lett.B336, 376 (1994). 
[10] L.D.Faddeev, Theor.Math.Phys.1, 1 (1970).

P.Senjanovich, Ann.Phys.(N.Y.)100, 277 (1976).

[11] R. Amorim and J. Barcelos-Neto, Phys. Lett. B333, 413 (1994); Phys. Rev. D53, 7129 (1996).

J. Barcelos-Neto and W. Oliveira, Int. J. Mod. Phys.A, vol.12, no.29, 5209 (1997).

[12] N. Banerjee, R. Banerjee, and S. Ghosh, Phys. Rev.D49, 1996 (1994).

[13] R. Banerjee and J. Barcelos-Neto, hep-th 9701080.

[14] J. Barcelos-Neto, Phys.Rev.D55, 2265 (1997).

[15] N. Banerjee and R. Banerjee, Mod. Phys. Lett. A11, 1919 (1996).

W.T. Kim, Y.-W. Kim, M.-I. Park and Y.-J. Park, J.Phys.G23, 325 (1997).

[16] R. Amorim and A. Das, Mod.Phys.Lett.A9, 3543 (1994).

R. Amorim, Z.Phys.C67, 695 (1995).

[17] G.S.Adkins,C.R.Nappi and E.Witten, Nucl.Phys.B228, 552 (1983). G.S.Adkins, Chiral Solitons, ed. Keh-Fei Liu, (World Scientific, 1987) p.99.

[18] T.D. Lee, Introduction to Field Theory and Particle Physics, (Harwood, New York, 1981) p.476.

[19] H.Verschelde, Nucl.Phys.A500, 573 (1989). Phys.Lett.B215, 444 (1988).

A.Toda, Prog.Theor.Phys.84, 324 (1990).

J.Ananias Neto, J.Phys.G21, 695 (1995). 
Z.Z.Israilov and M.M.Musakhanov, Int.J.Theor.Phys.30,9 1229 (1991).

K.Fujii, K.I.Sato, N.Toyota and A.P.Kobushkin, Phys.Rev.Lett.58,7 651 (1987). Phys.Rev.D35,6 1896 (1987).

B.Moussallam, Ann.Phys.(N.Y.) 225, 264 (1993).

B.Moussallam and D.Kalafatis, Phys.Lett.B272, 196 (1991).

G.Holzwarth, Phys.Lett.B291, 218 (1992), Nucl.Phys.A572, 69 (1994). 